Rev. Elev. Méd. vét. Pays trop., 1970, 23 (3) : 347-59

\title{
Action du Nitroxynil sur divers parasites du Zébu en Afrique centrale
}

\author{
par J. GUILHON, M. GRABER et E. BIRGI
}

\begin{abstract}
RESUME
Dans les régions tropicales sèches d'Afrique le Nitroxynil est un anthelminthique dont la polyvalence est limitée à Fasciola gigantica et à certains nématodes de l'abdomen et de l'intestin (Haemoncus, Bunostomum et Bosicola) parasites du zébu.

Les doses recommandées sont de $10 \mathrm{mg} / \mathrm{kg}$ pour les Fasciola déjà âgés de 12 semaines (de juillet à décembre au Tchad) et de $20 \mathrm{mg} / \mathrm{kg}$ pour les formes jeunes.

Les premiers accidents toxiques se manifestent à $50 \mathrm{mg} / \mathrm{kg}$.
\end{abstract}

Le Nytroxynil ou iodo-3 hydroxy-4 nitro-5 benzonitrile est un nouvel anthelminthique ( $\left.{ }^{1}\right)$ dont les propriétés fasciolicides ont été révélées en Angleterre par DAVIS et collab. et en France par l'un d'entre nous, en 1966.

Les résultats obtenus ultérieurement contre la fasciolose hépatique des ovins à Fasciola hepatica, en Europe, ont paru suffisamment encourageants pour entreprendre une étude plus approfondie de ce corps de façon à mieux déterminer l'étendue de son registre d'action sur les parasites du Zébu, en Afrique centrale (Tchad).

\section{MATERIEL ET METHODE}

Pour effectuer le travail commencé en décembre 1966 et terminé en juillet 1969, 77 zébus furent utilisés ( 29 femelles âgées et 48 bouvillons); 8 d'entre eux ont servi aux essais par voie buccale et 46 aux essais par voie sous-cutanée. Les lots furent répartis comme indiqué au tableau $\mathrm{I}$.

(1) Commercialisé sous les noms de Dovenix en France et de Trodax en Grande-Bretagne.
L'état des animaux était, dans l'ensemble, médiocre surtout à partir du mois de mars 1969. La saison des pluies précédente (1968) ayant été plus courte et faiblement hygrométrique, l'état des femelles âgées très maigres, souvent cachectiques, a permis d'apprécier plus utilement la toxicité du Nitroxynil à l'égard d'un bétail dont la résistance se trouvait très amoindrie du fait d'une alimentation réduite.

Les nombreux helminthes recueillis à l'autopsie des animaux utilisés sont indiqués par groupe zoologique (tableau II).

\section{Protocole}

\section{A) Avant le traitement}

Chaque animal a été mis en observation pendant 48 heures et des examens coproscopiques (méthode de sédimentation de BRUMPT) ont été effectués pour apprécier l'importance exacte du parasitisme, surtout en ce qui concerne les Trématodes et les Nématodes.

\section{B) Traitement}

La totalité des essais a été réalisée sans diète préalable car le traitement sur le terrain, du fait de la mentalité des éleveurs, ne doit com- 
Retour au menu

TABLEAU $N^{*} \mathrm{I}$

Les animaux utilisés

\begin{tabular}{|c|c|c|c|c|}
\hline & \multicolumn{2}{|c|}{ Vole buccale } & \multicolumn{2}{|c|}{ Vole sous-cutanée } \\
\hline & Bouvillons & $\begin{array}{c}\text { Feme11es } \\
\text { âgëes }\end{array}$ & Bauvilions & $\begin{array}{c}\text { Femelles } \\
\text { âgés }\end{array}$ \\
\hline $\begin{array}{l}\text { Essais thérapeutiques sur } \\
\text { Fascioles adultes }\end{array}$ & 3 & 5 & 21 & 13 \\
\hline $\begin{array}{l}\text { Essais thërapeutiques sur } \\
\text { Fascioles immatures }\end{array}$ & & & 6 & 6 \\
\hline Tëmoins & & & 13 & 4 \\
\hline Essais d'1ntoxication & & & 5 & 1 \\
\hline
\end{tabular}

TABLEAU N"II

Espèces parastes et nombre d'anlmaux atteints

\begin{tabular}{|c|c|c|c|}
\hline \multirow{2}{*}{ Espềces parasites } & \multicolumn{2}{|c|}{ Nombre d'animaux parasitês } & \multirow{2}{*}{ T o t a 1} \\
\hline & Adultes & Bouvillons & \\
\hline \multicolumn{4}{|l|}{ Trématodes } \\
\hline Dicrocoeliwn hospes & - & 19 & 19 \\
\hline Easciola gigantiaa & 13 & 24 & 37 \\
\hline Cotyzophoron coty Lophorum & - & 10 & 10 \\
\hline Paramphistomum miarobotinium & 11 & 2 & 13 \\
\hline Conmyerius spatiosus & 1 & - & 1 \\
\hline Carmyerius graberi & 1 & - & 1 \\
\hline Camperius parvipopizzatus & 1 & - & 1 \\
\hline Schistosoma bovis & 17 & 5 & 22 \\
\hline \multicolumn{4}{|l|}{ Cestodes } \\
\hline Thysconiezia ovizia & 1 & 4 & 5 \\
\hline Moniezia benedeni & 1 & 3 & 4 \\
\hline oysticercus bovis & 1 & 4 & 5 \\
\hline Echinococcus polymorphus & 3 & - & 3 \\
\hline \multicolumn{4}{|l|}{ Nématodes } \\
\hline Strongyzoides papizlosus & - & 1 & 1 \\
\hline Bosicola radiatum & 8 & 14 & 22 \\
\hline Bunostomum phlebotomum & - & 13 & 13 \\
\hline $\begin{array}{l}\text { Cooperia punotata ) } \\
\text { Cooperia pectinata) }\end{array}$ & 12 & 26 & 38 \\
\hline Hoemoncus contortus & 1 & 9 & 10 \\
\hline Setaria Labiato popillosa & 14 & 6 & 20 \\
\hline Onchocerca gutturosa & 17 & 11 & 28 \\
\hline Onchocerca armizlata & 15 & 2 & 17 \\
\hline Trichuris globutosa & - & 4 & 4 \\
\hline
\end{tabular}

porter aucune préparation particulière de l'animal. Le médicament a été administré par la voie individuelles, cimentées. sous-cutanée ou par la voie buccale, et les zébus après marquage ont été placés dans des stalles, 
TABLEAU N'III

Témoins : espèces parasites et nombre d'animaux atteints.

\begin{tabular}{|c|c|c|c|c|c|c|}
\hline \multirow{2}{*}{ Helminthes en cause } & \multicolumn{3}{|c|}{$\begin{array}{c}\text { Nombre d'animaux } \\
\text { parasités }\end{array}$} & \multicolumn{3}{|c|}{$\begin{array}{c}\text { Paids }(x) \text { ou nombre } \\
\text { de parasites } \\
\text { (moyenne) }\end{array}$} \\
\hline & A & B & C & $\mathbf{A}$ & $\mathrm{B}$ & $\mathrm{C}$ \\
\hline Diorocoeliwm hospes & 3 & 4 & - & 4 & 4 & - \\
\hline Easoiola gigantica & 2 & 4 & 4 & 21 & 5 & 14 \\
\hline Paramphistomm microbothrium & - & $\sim$ & 3 & - & - & 26 \\
\hline Carmyerius parvipapizlatus & - & - & $\mathbf{1}$ & - & - & 0,8 \\
\hline Cotyiophoron cotylophorum & - & 3 & - & - & 1 & - \\
\hline Schistosoma bovis & - & - & 7 & - & - & 21 \\
\hline Thysanieaia ovilla & - & - & 3 & - & - & 7.5 \\
\hline Monieaia benedeni & - & - & 1 & - & - & 8 \\
\hline Custicercus bovis & 1 & - & - & 1 & - & - \\
\hline Bunostomom phlebotomum & 3 & 1 & 2 & 19 & 22 & 5 \\
\hline Bosicola radiatw & 2 & - & 1 & 33 & - & 85 \\
\hline $\begin{array}{l}\text { Cooperia punctata }) \\
\text { Cooperia peotinata }\end{array}$ & 3 & - & 3 & 105 & - & 3300 \\
\hline Haemonous contortus & - & - & 2 & - & - & 335 \\
\hline Setaria labiato papizlosa & 2 & - & 1 & 4 & - & 1 \\
\hline Onchocerca armiliata & - & - & 1 & - & - & - \\
\hline Onchocerca gutturosa & 1 & - & 2 & - & - & - \\
\hline Trichuris globulosa & 2 & - & - & 3 & - & - \\
\hline
\end{tabular}

(x) moyenne du polds (en grammes) pour les Cestodes, Paramphistomidés et les Gabtrothylacidés seulement.

\section{C) Après le traitement}

1. Pour chaque sujet, il a été procédé pendant 8 jours : liers;

- à des examens coproscopiques journa-

- au ramassage des fèces trois fois par jour et à leur examen minutieux à l'œil nu, dans le but de détecter les Trématodes, les Cestodes et les Nématodes expulsés qui ont été recueillis, pesés ( ${ }^{2}$ ) ou comptés $\left({ }^{3}\right)$, et formolés.

2. Les animaux furent ensuite sacrifiés et autopsiés. Une dernière série d'examens coproscopiques a été faite le jour même de leur mort.

Les parasites demeurés dans le foie, la vésicidès.

(2) Cestodes, Paramphistomidés et Gastrothyla-

(3) Nématodes, Schistosomes et Fascioles. cule biliaire ou l'intestin ont été récoltés ou pesés.

Pour les fascioles et les paramphistomes il a été tenu compte de leur aspect extérieur, de leur survie éventuelle et de leur état de maturité (sur coupes colorées et après écrasement de lutérus entre deux lames).

Par ailleurs, la muqueuse duodénale a été grattée sur $30-60 \mathrm{~cm}$ et les prélèvements, placés entre lame et lamelle ont été examinés de manière à mettre en évidence Cooperia et Strongyloides papillosus.

D) Les résultats sont appréciés par comparaison :

- entre le nombre d'œufs au gramme, avant, pendant et après le dernier jour du traitement;

- entre le nombre de parasites éliminés après le traitement et le nombre de parasites retrouvés à l'autopsie;

- avec l'infestation des témoins. 
Pour obtenir les fascioles immatures, les bouvillons ont été infestés avec un nombre variable de métacercaires âgées de 7 à 13 jours .

Ils ont été traités ultérieurement au Nitroxynil $40,51,54-66,64-81,73-85$ et 84 jours après leur infestation expérimentale, à des doses variables.

L'autopsie fut effectuée 4 jours après l'administration du médicament. L'état de survie des jeunes fascioles a été observé dans de l'eau tiède ou dans la bile de bœuf à $39^{\circ} \mathrm{C}$, à la loupe.

Par contre les Distomes de moins de 50 jours, tués par l'anthelminthique se comportent comme des corps étrangers et un nodule réactionnel se forme rapidement autour d'eux. Le parasite est alors opaque et replié sur lui-même. S'il est encore vivant il est blanchâtre, clair, et apparaît en extension dans le liquide de fixation. La loupe permet d'observer les mouvements des jeunes parasites extraits à proximité de la capsule de Glisson et placés dans de l'eau tiède. Mais les réactions sont très fugaces et demandent une observation attentive.

Chaque animal infesté artificiellement a pu être comparé à un témoin ayant reçu le même nombre de métacercaires que lui. 17 animaux ont servi à cet usage, répartis de la façon suivante en fonction de l'époque des interventions et de la richesse de leur infestation :

- Printemps 1967 (A) : 4 bouvillons (Bouar) ;

- Printemps 1968 (B) : 5 bouvillons (Bouar);

- Printemps 1969 (C) : 4 bouvillons (Fort-Lamy) - 4 femelles (Massakory). (Tableau III)

\footnotetext{
TABLEAU $\mathrm{N}^{*} \mathrm{IV}$

Examens coproscoplques - Rësultats globaux

(Moyenne du nombre d'oeufs au gramme de matière fécale)
}

\begin{tabular}{|c|c|c|c|c|c|c|}
\hline \multirow[t]{2}{*}{$\begin{array}{c}\text { Doses } \\
\left(\mathrm{mg} / \mathrm{kg}_{\mathrm{g}}\right)\end{array}$} & \multicolumn{2}{|c|}{ Avant traitement } & \multicolumn{2}{|c|}{ Après traitement } & \multicolumn{2}{|c|}{$\begin{array}{l}10 \text { fours après le } \\
\text { traitement }(x)\end{array}$} \\
\hline & 1 & 2 & 1 & 2 & 1 & 2 \\
\hline 10 & - & 23 & - & 8 & - & 0 \\
\hline 20 & - & 17 & - & 37 & - & 52 \\
\hline 30 & - & 21 & - & 52 & - & 157 \\
\hline 40 & 0 & - & 0 & - & 0 & - \\
\hline
\end{tabular}

1 : voie buccale

2 : voie sous-cutanée

(x) de 3 a 10 jours après $I^{\prime}$ admintstration de l'anthelminthique.

TABLEAU N"V

Dicrocoelium hoapes adultes - Résultats des autopsies.

\begin{tabular}{|c|c|c|c|c|c|c|c|c|}
\hline \multirow{2}{*}{$\begin{array}{c}D \circ g \text { e s } \\
(\mathrm{mg} / \mathrm{kg})\end{array}$} & \multicolumn{2}{|c|}{10} & \multicolumn{2}{|c|}{20} & \multicolumn{2}{|c|}{30} & \multicolumn{2}{|c|}{40} \\
\hline & 1 & 2 & 1 & 2 & 1 & 2 & 1 & 2 \\
\hline Nombre d'anlmaux utilisēs & - & 4 & - & 4 & - & 2 & 2 & - \\
\hline $\begin{array}{l}\text { Nombre d'antmaux totalement } \\
\text { déparasités }\end{array}$ & - & 0 & - & 0 & - & 0 & 0 & - \\
\hline $\begin{array}{l}\text { Nombre total de Dicrocoetion } \\
\text { morts a } 1 \text { 'autopsie }\end{array}$ & - & 0 & - & 2 & - & 0 & 0 & - \\
\hline $\begin{array}{l}\text { Nombre total de Diarocoelizm } \\
\text { vivants à l'autopsie (x) }\end{array}$ & - & 219 & - & 186 & - & 192 & 120 & - \\
\hline Efflcacité & \multicolumn{2}{|c|}{ Nu11e } & - & $1 \mathrm{p} \cdot 100$ & \multicolumn{2}{|c|}{ Nulle } & \multicolumn{2}{|c|}{ Nul1e } \\
\hline
\end{tabular}

(x) dans de la blle de boeuf à $39^{\circ} \mathrm{C}$. 


\section{RESULTATS}

Les résultats obtenus dans les conditions expérimentales précisées sont répartis dans plusieurs tableaux par groupe zoologique d'helminthes (Trématodes, Cestodes, Nématodes) et dans chacun d'entre eux par famille (Paramphistomidés, Gastrothylacidés, Schistosomidés) ou par espèce.

\section{A. Action sur les Trématodes (Tableaux IV, V)}

\section{Dicrocoelium hospes adultes}

Le Nitroxynil, jusqu'à $30 \mathrm{mg}$ en injection sous-cutanée, n'agit pas sur Dicrocoelium hospes des canaux biliaires. Il en est de même de la dose de $40 \mathrm{mg} / \mathrm{kg}$ par la bouche.

2. Fasciola gigantica adultes

(Tableaux VI, VII)

TABLEAU $N^{*} \mathrm{VI}$

Fasciola gigantica adultes - Examens coproscopiques

(Moyenne du nombre d'oeufs au granme de matière fêcale)

\begin{tabular}{|c|c|c|c|c|c|c|}
\hline \multirow[t]{2}{*}{$\begin{array}{l}\text { Doses } \\
(\mathrm{mg} / \mathrm{kg})\end{array}$} & \multicolumn{2}{|c|}{ Avant traltement } & \multicolumn{2}{|c|}{ Après traltement } & \multicolumn{2}{|c|}{$\begin{array}{l}10 \text { jours après le } \\
\text { traltetnent }(x)\end{array}$} \\
\hline & 1 & 2 & 1 & 2 & 1 & 2 \\
\hline 10 & - & 60 & - & 33 & - & 0 \\
\hline $20^{x x}$ & - & 0 & - & 0 & - & 0 \\
\hline 30 & - & 42 & - & 0 & - & 0 \\
\hline 40 & 35 & - & 0 & - & 0 & - \\
\hline $100^{x \times x}$ & 0 & - & 0 & - & 0 & - \\
\hline $125^{x \times x}$ & 0 & - & 0 & - & 0 & - \\
\hline
\end{tabular}

(x) de 3 à 10 jours plus tard; (xx) Fasoiola Immatures; (xxx) Fasciola mûres, mals en très pettes nombres.

TABLEAU N*VII

Fasciola adultes - Résultats des autopsies

\begin{tabular}{|c|c|c|c|c|c|c|}
\hline$\underset{(\mathrm{mg} / \mathrm{kg})}{\mathrm{D}}$ & $\begin{array}{r}10 \\
2\end{array}$ & $\begin{array}{r}20 \\
2\end{array}$ & $\begin{array}{r}30 \\
2\end{array}$ & $\begin{array}{r}40 \\
1\end{array}$ & $\begin{array}{c}100 \\
1\end{array}$ & $\begin{array}{c}125 \\
1\end{array}$ \\
\hline Nombre d'enimaux utiligés & 7 & 3 & 1 & 2 & 1 & 1 \\
\hline $\begin{array}{l}\text { Nombre d'animiux totalement } \\
\text { deparas } 1 \text { tes }\end{array}$ & 7 & 3 & 1 & 1 & 1 & 1 \\
\hline $\begin{array}{l}\text { Nombre de Fascioles vivantes } \\
\text { a l'autopsie }\end{array}$ & 0 & 0 & 0 & 1 & 0 & 0 \\
\hline $\begin{array}{l}\text { Nombre de Fascloles morte: } \\
\text { à } 1^{\prime} \text { autopsie } \\
\text { Mûre: } \\
\text { Immecures }\end{array}$ & $\begin{array}{r}18 \\
-\end{array}$ & - & $\begin{array}{l}0 \\
0\end{array}$ & $\begin{array}{l}0 \\
0\end{array}$ & $\begin{array}{l}3 \\
0\end{array}$ & 1 \\
\hline $\begin{array}{l}\text { Nombre d'animaux prétentant des } \\
\text { oeufs et des lésiona a } 1 \text { 'autopale }\end{array}$ & 7 & 0 & 1 & 1 & & \\
\hline $\begin{array}{l}\text { Nombre d'animaux ne présentant } \\
\text { pas d'oeufs, mals des léstons } \\
\text { à l'autopele }\end{array}$ & - & 3 & - & - & & \\
\hline Efficacité & totale & totale & totale & $\begin{array}{c}1 \\
\operatorname{sur} \\
2\end{array}$ & totale & totale \\
\hline
\end{tabular}


TABLEAU $N^{\circ}$ VIII

Résultats des autopsies

\begin{tabular}{|c|c|c|c|c|c|c|}
\hline $\begin{array}{c}\text { Bouvillons } \\
\mathrm{N}^{\bullet}\end{array}$ & $\begin{array}{c}\text { Nombre de } \\
\begin{array}{c}\text { Métacercaires } \\
\text { reçues }\end{array} \\
\end{array}$ & $\begin{array}{r}\text { Doses } \\
\text { mg } / \mathrm{kg} \\
\mathrm{S}-\mathrm{C} \\
\end{array}$ & $\begin{array}{c}\text { Age } \\
\text { des } \\
\text { douves }\end{array}$ & $\begin{array}{l}\text { Nombre de } \\
\text { douves a } \\
1 \text { 'autopsie }\end{array}$ & Efflcactié & Témains \\
\hline 37 & 500 & 20 & 40 jours & 4 mortes & Totale & $\begin{array}{c}N^{\circ} 33 \\
\text { Métacercaires : } 500 \\
\text { Douves : } 47\end{array}$ \\
\hline 38 & 500 & 15 & 51 jours & $\begin{array}{l}35 \text { mortes } \\
17 \text { vivantes }\end{array}$ & 67,3 p. 100 & $\begin{array}{c}\mathbb{N}^{\bullet} 35 \\
\text { Métacerca1res : } 500 \\
\text { Douves : } 173\end{array}$ \\
\hline 3 & $1.000^{+}$ & 40 & $\begin{array}{l}54-66 \\
\text { jours }\end{array}$ & 92 mortes & Totale & $\begin{array}{c}N^{\circ} 1 \\
\text { Métacezcaires : } 1.000 \\
\text { Douves : } 225\end{array}$ \\
\hline 8 & $2,000^{+}$ & 20 & $\begin{array}{l}64-81 \\
\text { jours }\end{array}$ & 344 mortes & Totale & $\begin{array}{c}N^{0} 9 \\
\text { Métacercaires : } 2.000 \\
\text { Douves : } 734\end{array}$ \\
\hline 15 & $5.000^{+}$ & 20 & $\begin{array}{l}73-85^{\circ} \\
\text { jours }\end{array}$ & 1.186 mortes & Totale & $\begin{array}{c}\mathrm{N}^{\bullet} 16 \\
\text { Mëtacercaires }: 5.000 \\
\text { Douves }: 1.172\end{array}$ \\
\hline 31 & 500 & 10 & 84 jours & 79 mortes & Totale & $\begin{array}{c}\mathrm{N}^{\mathrm{a}} 32 \\
\text { Métacercaires : } 500 \\
\text { Douves : } 213\end{array}$ \\
\hline
\end{tabular}

+ en 2 fols 12 jours d'intervalle.

Le Nitroxynil, à la dose unique de $10 \mathrm{mg} / \mathrm{kg}$, est capable non seulement de détruire les fascioles mûres en instance de ponte, c'est-à-dire celles qui, dans les conditions du Tchad, sont âgées de $97-105$ jours et plus, mais aussi celles de 12 semaines.

La dose de $15 \mathrm{mg} / \mathrm{kg}$ ne tue que les deux tiers des parasites âgés d'environ 7 semaines.
Les fascioles de 40 jours ne sont éliminées qu'à la dose de $20 \mathrm{mg} / \mathrm{kg}$ administrée par voie sous-cutanée.

Ainsi que le laissent prévoir les résultats indiqués dans le tableau VIII, la dose de $10 \mathrm{mg} / \mathrm{kg}$ peut être préconisée contre les Fascioles de plus de 12 semaines. La dose doit être doublée (20 $\mathrm{mg} / \mathrm{kg}$ ) pour éliminer les douves plus jeunes.

TABLEAU $\mathrm{N}^{\bullet}$ IX

Examens coproscopiques - Rẽsultats globaux

(Moyenne du nombre d'oeufs au gramme de mattère fécale)

\begin{tabular}{|c|c|c|c|c|c|c|}
\hline \multirow{2}{*}{$\begin{array}{c}\text { Do s es } \\
(\mathrm{mg} / \mathrm{kg})\end{array}$} & \multicolumn{2}{|c|}{ Avant traitement } & \multicolumn{2}{|c|}{ Après traitement } & \multicolumn{2}{c|}{$\begin{array}{c}\text { jours après le } \\
\text { traitement (x) }\end{array}$} \\
\cline { 2 - 6 } & 1 & 2 & 1 & 2 & 1 & 2 \\
\hline 10 & - & 2,6 & - & 9,7 & - & 0 \\
20 & - & 0 & - & 0 & - & 0 \\
30 & - & 0 & - & 0 & - & 0 \\
40 & 0 & - & 0 & - & 0 & - \\
50 & 315 & - & 141 & - & 0 & - \\
75 & 210 & - & 175 & - & 0 & - \\
100 & 157 & - & 0 & - & 0 & - \\
\hline
\end{tabular}

(x) de 3 à 10 jours aptès 1 'administration de l'anthelminthique. 
TABLEAU $\mathrm{N}^{\circ} \mathrm{X}$

Résultats des autopsies

\begin{tabular}{|c|c|c|c|c|c|c|c|c|}
\hline $\begin{array}{c}\text { Dos e s } \\
(\mathrm{mg} / \mathrm{kg})\end{array}$ & $\begin{array}{c}10 \\
1-2\end{array}$ & $\begin{array}{l}20 \\
2\end{array}$ & $\begin{array}{r}30 \\
2\end{array}$ & $\begin{array}{r}40 \\
1\end{array}$ & $\begin{array}{r}50 \\
1\end{array}$ & $\begin{array}{r}60 \\
2\end{array}$ & $\begin{array}{r}75 \\
1\end{array}$ & $\begin{array}{r}100 \\
1\end{array}$ \\
\hline Nombre d'animaux ut111séb & 9 & 1 & 3 & 1 & 1 & 1 & 1 & 2 \\
\hline $\begin{array}{l}\text { Nombre d'antmaux totalement } \\
\text { déparasités }\end{array}$ & 0 & 0 & 0 & 0 & 0 & 0 & 0 & 0 \\
\hline $\begin{array}{l}\text { Parasites morts à l'autopsie } \\
\text { (en grammes) }\end{array}$ & 0 & 0 & 0 & 0 & 0 & 0 & 115 & 0 \\
\hline $\begin{array}{l}\text { Parasités vivants à l'autopsie } \\
\text { (en grammes) }\end{array}$ & 227,2 & 0,1 & 4 & 4 & 138 & 2 & 5,5 & 143 \\
\hline Efficact tê & Nulle & Nulle & Nulle & Nulle & Nulle & Nulle & 21 p. 100 & Nulle \\
\hline
\end{tabular}

4. Action sur les Paramphistomidés ( $P$. microbothrium, Cotylophoron cotylophorum) et sur les Gastrothylacidés (Carmyerius spatiosus, C. graberi, C. parvipapillatus). (Tableaux IX, $\mathrm{X})$.

Le Nitroxynil, que ce soit par voie buccale ou par la voie sous-cutanée, est pratiquement dépourvu de tout pouvoir anthelminthique à l'égard des Paramphistomidés et des Gastrothylacidés parasites de la panse.

\section{Schistosoma bovis et Schistosoma mattheei} (veines mésentériques) (Tableau XI).

TABLEAU $\mathrm{N}^{\circ} \mathrm{XI}$

Rẻsultats des autopsies

\begin{tabular}{|c|c|c|c|c|c|c|}
\hline $\begin{array}{l}D \circ s \text { e s } \\
(\mathrm{mg} / \mathrm{kg})\end{array}$ & $\begin{array}{r}10 \\
2\end{array}$ & $\begin{array}{r}40 \\
2\end{array}$ & $\begin{array}{r}60 \\
2\end{array}$ & $\begin{array}{r}75 \\
1\end{array}$ & $\begin{array}{r}100 \\
1\end{array}$ & $\begin{array}{r}125 \\
1\end{array}$ \\
\hline Nombre d'animaux parasités & 9 & 1 & 1 & 1 & 2 & 1 \\
\hline $\begin{array}{l}\text { Nombre d'animaux totalement } \\
\text { déparasités }\end{array}$ & 0 & 0 & 0 & 0 & 0 & 0 \\
\hline $\begin{array}{l}\text { Nombre total de parasites } \\
\text { encore vivanto à l'autopsie }\end{array}$ & 159 & 8 & 7 & 21 & 32 & 10 \\
\hline Efficacité & Nu1le & Nulle & Nulle & Nulle & Nulle & Nulle \\
\hline
\end{tabular}

\section{B. Action sur les Cestodes}

1. Moniezia benedeni (Intestin) (Tableau XII)

TABLEAU $\mathrm{N}^{\circ} \mathrm{XII}$

Résultats des autopsies

\begin{tabular}{|c|c|c|c|}
\hline \multicolumn{1}{|c|}{$\begin{array}{c}\text { Do s e s } \\
\text { (mg/kg) }\end{array}$} & $\begin{array}{r}10 \\
2\end{array}$ & $\begin{array}{r}30 \\
2\end{array}$ & $\begin{array}{r}60 \\
1\end{array}$ \\
\hline Nombre d'animaux ut1lisês & 1 & 1 & 1 \\
\hline $\begin{array}{l}\text { Nombre d'animaux totalement } \\
\text { dêparasités }\end{array}$ & 0 & 0 & 0 \\
\hline $\begin{array}{l}\text { Poids des Cestodes évacuês } \\
\text { (en grammes) }\end{array}$ & 0 & 0 & 0 \\
\hline $\begin{array}{l}\text { Présence ou absence (-) de } \\
\text { Cestodes à 1'autopsie }\end{array}$ & 5 & 4 & 5 \\
\hline
\end{tabular}

2. Thysaniezia Ovilla (Intestin) (Tableau XIII)

TABLEAU $N^{\circ}$ XIII

Résultats des autopsies

\begin{tabular}{|c|c|c|}
\hline $\begin{array}{l}\text { D os e s } \\
(\mathrm{mg} / \mathrm{kg})\end{array}$ & $\begin{array}{r}50 \\
1\end{array}$ & $\begin{array}{r}60 \\
2\end{array}$ \\
\hline Nombre d'animaux ut1lisés & 1 & 1 \\
\hline $\begin{array}{l}\text { Nombre d'animaux totalement } \\
\text { dëparasités }\end{array}$ & 0 & 0 \\
\hline $\begin{array}{l}\text { Polds de Cestoder évacués } \\
\text { (en grammes) }\end{array}$ & 0 & 0 \\
\hline $\begin{array}{l}\text { Présence ou absence (-) de } \\
\text { Cestodes à l'autopsie }\end{array}$ & 1 & 20 \\
\hline
\end{tabular}


3. Cysticercus bovis (muscles) (Tableau XIV)

Comme dans le cas de parasitisme par $M$. benedeni et par $T$. ovilla, le Nitroxynil est sans effet sur Cysticercus bovis.

\section{Action sur les Nématodes}

1. Examens coproscopiques (Tableau XV)
TABLEAU $\mathbb{N}^{\circ} \mathrm{XIV}$

Résultats des autopsies

\begin{tabular}{|c|c|c|}
\hline Doses mg/kg (S-C) & 10 & 30 \\
\hline Nombre d'animaux utilisẻs & 3 & 1 \\
\hline $\begin{array}{l}\text { Nombre d'antmaux totalement } \\
\text { déparasités }\end{array}$ & 0 & 0 \\
\hline $\begin{array}{l}\text { Nombre de Cysticerques } \\
\text { encore vivants (x) }\end{array}$ & 153 & 3 \\
\hline
\end{tabular}

(x) après évagination dans de la blle à $+39^{\circ} \mathrm{C}$ de 4 à 5 jours après le traittement.

\section{TABLEAU $\mathrm{N}^{\bullet} \mathrm{XV}$ \\ Résultats globaux}

"Strongles" (Haemoncus, Cooperia, Bosicola et Bunostomu) Moyenne du nombre d'oeufs au gramme de matière fécale.

\begin{tabular}{|c|c|c|c|c|c|c|}
\hline \multirow{2}{*}{$\begin{array}{c}\text { Doses } \\
\mathrm{mg} / \mathrm{kg}\end{array}$} & \multicolumn{2}{|c|}{ Avant traitement } & \multicolumn{2}{|c|}{ Après traftement } & \multicolumn{2}{c|}{$\begin{array}{c}\text { jours après le } \\
\text { tra1 tement }(\mathrm{x})\end{array}$} \\
\cline { 2 - 7 } & 1 & 2 & 1 & 2 & 1 & 2 \\
\hline 10 & - & 140 & - & 26 & - & 14 \\
15 & - & 105 & - & 24 & - & 0 \\
20 & - & 114 & - & 28 & - & 0 \\
30 & - & 94 & - & 16 & - & 0 \\
40 & 157 & - & 31 & - & 105 & - \\
50 & 52 & - & 157 & - & 105 & - \\
60 & 840 & - & 126 & - & 0 & - \\
75 & 472 & - & 21 & - & 0 & - \\
100 & 180 & - & 42 & - & 105 & - \\
\hline
\end{tabular}

(x) de 4 à 10 jours après l'administration de 1 'anthelminthique

2. Bosicola radiatum (Cæcum) -

Adultes mûrs (Tableau XVI)

TABLEAU N $N^{\circ}$ XVI

Résultats des autopsies

\begin{tabular}{|c|c|c|c|c|c|c|c|c|c|}
\hline $\begin{array}{l}\text { Doses } \\
(\mathrm{mg} / \mathrm{kg})\end{array}$ & $\begin{array}{r}10 \\
2\end{array}$ & $\begin{array}{r}15 \\
2\end{array}$ & $\begin{array}{r}20 \\
2\end{array}$ & $\begin{array}{r}30 \\
2\end{array}$ & $\begin{array}{r}40 \\
1\end{array}$ & $\begin{array}{r}50 \\
1\end{array}$ & $\begin{array}{l}60 \\
1\end{array}$ & $\begin{array}{r}75 \\
1\end{array}$ & $\begin{array}{r}100 \\
1\end{array}$ \\
\hline Nombre d'an1maux ut111sés & 7 & 2 & 2 & 1 & 1 & 1 & 1 & 1 & 1 \\
\hline $\begin{array}{l}\text { Nombre d'animaux totalement } \\
\text { déparas } 1 \text { tês }\end{array}$ & 7 & 1 & 1 & 1 & 0 & 0 & 0 & 0 & 0 \\
\hline Nombre de parasites évacués & 83 & 1 & 3 & 40 & 0 & 0 & 0 & 0 & 9 \\
\hline $\begin{array}{l}\text { Nombre de parasites recueillis } \\
\text { à } l^{\prime} \text { autopste }\end{array}$ & 1 & 0 & 3 & 0 & 20 & 60 & 6 & 110 & 24 \\
\hline Efflcacité & $98, \mathrm{Bp}, 100$ & Totale & $95 \mathrm{p} .100$ & Totale & Nulle & Nulle & Nulle & Nulle & $27 \mathrm{p} .100$ \\
\hline
\end{tabular}




\section{Bunostomum phlebotomum}

adultes (duodénum) (Tableau XVII)

TABLEAU $\mathrm{N}^{\circ}$ XVII

Résultats des autopstes

\begin{tabular}{|c|c|c|c|c|c|}
\hline$\underset{(\mathrm{mg} / \mathrm{kg})}{\mathrm{D}}$ & $\begin{array}{r}10 \\
2\end{array}$ & $\begin{array}{r}15 \\
2\end{array}$ & $\begin{array}{r}20 \\
2\end{array}$ & $\begin{array}{r}50 \\
2\end{array}$ & $\begin{array}{r}60 \\
1\end{array}$ \\
\hline Nombre d'animaux utilisês & 2 & I & 2 & 1 & 1 \\
\hline $\begin{array}{l}\text { Nombre d'animaux totalement } \\
\text { déparasitês }\end{array}$ & 2 & I & 2 & 1 & 0 \\
\hline Nombre de parasites évacués & 78 & 1 & 20 & 4 & 0 \\
\hline $\begin{array}{l}\text { Nombre de parasites recueillis } \\
\text { a l'autopsie }\end{array}$ & 0 & 0 & 0 & 0 & 8 \\
\hline Efflcacitë & Totale & Totale & Totale & Totale & Nulle \\
\hline
\end{tabular}

\section{Cooperia punctata et Cooperia pectinata} adultes (intestin grêle) (Tableau XVIII)

TABLEAU $N^{\circ}$ XVIII

Résultats des autopsies

\begin{tabular}{|c|r|c|c|c|c|c|c|c|}
\hline $\begin{array}{c}\text { Do s e s } \\
\text { (ng/kg) }\end{array}$ & $\begin{array}{r}10 \\
2\end{array}$ & $\begin{array}{r}15 \\
2\end{array}$ & $\begin{array}{r}20 \\
2\end{array}$ & $\begin{array}{r}30 \\
2\end{array}$ & $\begin{array}{r}40 \\
1\end{array}$ & $\begin{array}{r}60 \\
1\end{array}$ & $\begin{array}{r}75 \\
1\end{array}$ & $\begin{array}{r}100 \\
1\end{array}$ \\
\hline Nombre d'an1maux vitllisés & 16 & 2 & 6 & 3 & 1 & 1 & 1 & 2 \\
\hline $\begin{array}{l}\text { Nombre d'animaux totalement } \\
\text { déparasités }\end{array}$ & 0 & 0 & 0 & 0 & 0 & 0 & 0 & 0 \\
\hline $\begin{array}{l}\text { Nombre total de parasites } \\
\text { retrouvés à 1'autopsie }\end{array}$ & 546 & 85 & 476 & 60 & 30 & 1 & 142 & 4.867 \\
\hline
\end{tabular}

\section{Haemoncus contortus (caillette)}

\section{(Tableau XIX)}

TABLEAU $\mathrm{N}^{\circ} \mathrm{XIX}$

Résultats des autopsles

\begin{tabular}{|c|c|c|c|c|c|c|}
\hline $\begin{array}{c}\text { Do s e s } \\
(\mathrm{mg} / \mathrm{kg})\end{array}$ & $\begin{array}{r}10 \\
2\end{array}$ & $\begin{array}{r}15 \\
2 \\
\end{array}$ & $\begin{array}{r}20 \\
2\end{array}$ & $\begin{array}{r}40 \\
1\end{array}$ & $\begin{array}{r}50 \\
2\end{array}$ & $\begin{array}{c}60 \\
1\end{array}$ \\
\hline Nombre d'anfuaux ut1lisés & 1 & 2 & 1 & 1 & 1 & 1 \\
\hline $\begin{array}{l}\text { Nombre d'animaux totalement } \\
\text { déparasttês }\end{array}$ & 1 & 2 & 1 & 0 & 0 & 1 \\
\hline Nombre de parasites évacués & 9 & 10 & 0 & 0 & 0 & 5 \\
\hline $\begin{array}{l}\text { Nombre de parasites recueillis } \\
\text { à 1'autops le }\end{array}$ & 0 & 0 & 10 & 9 & 25 & 0 \\
\hline Efflcacité & $\begin{array}{l}\text { semble } \\
\text { totale }\end{array}$ & $\begin{array}{l}\text { semble } \\
\text { totale }\end{array}$ & partielle & Nulle & Nulle & $\begin{array}{l}\text { semble } \\
\text { totale }\end{array}$ \\
\hline
\end{tabular}

1: voie buccale; 2 : voie sous-cutanêe.

$x=d i m i n u t i o n$ du nombre d'oeufs et de larves $L_{3}$ à la coproculture. 
6. Trichuris globulosa (cæcum) (Tableau XX)

TABLEAU $\mathrm{N}^{\circ} \mathrm{XX}$

Résultats sur autopsie

\begin{tabular}{|l|c|c|}
\hline \multicolumn{1}{|c|}{$\begin{array}{c}\text { D o s e s } \\
\text { (mg/kg) }\end{array}$} & $\begin{array}{r}10 \\
2\end{array}$ & $\begin{array}{r}20 \\
2\end{array}$ \\
\hline Nombre d'anImaux utılisés & 2 & 1 \\
\hline $\begin{array}{l}\text { Nombre d'animaux } \\
\text { totalement déparasités }\end{array}$ & 0 & 0 \\
\hline $\begin{array}{l}\text { Nombre de parasites } \\
\text { évacuếs }\end{array}$ & 0 & 0 \\
\hline $\begin{array}{l}\text { Nombre de parasites } \\
\text { recueillis à l'autopsie }\end{array}$ & 9 & 1 \\
\hline Efficacité & Nulle & Nulle \\
\hline
\end{tabular}

\section{Filaires diverses}

Certaines constatations avaient pu laisser supposer que le Nitroxynil pouvait agir sur plusieurs Filaires du Zébu rencontrées au Tchad: Setaria labiato-papillosa du péritoine, Onchocerca armillata de l'aorte et Onchocerca gutturosa du ligament cervical. Au bout de 10 jours, plusieurs de ces parasites montraient des cuticules ramollies et éclatées. Une comparaison établie avec des témoins non traités montre en définitive que le Nitroxynil n'a aucune action sur les Filaires des ligaments, des vaisseaux et des séreuses.

\section{DISCUSSION GENERALE}

Le Nitroxynil, par la voie parentérale, se comporte comme un fasciolicide. A la dose de $10 \mathrm{mg} / \mathrm{kg}$, les fascioles (Fasciola gigantica) âgées de 12 semaines et plus sont tuées par l'anthelminthique. L'élimination des douves plus jeunes (40 à 80 jours) exige l'emploi d'une dose double $(20 \mathrm{mg} / \mathrm{kg})$.

Ces résultats sont assez semblables à ceux qui ont été obtenus en Europe sur Fasciola hepatica.

Au contraire, le médicament administré toujours par la même voie sous-cutanée est totalement dépourvu d'efficacité à l'égard de Dicro- coelium hospes des canaux biliaires, des Paramphistomidés et des Gastrothylacidés fréquents dans la panse, de Schistosoma bovis, des grands Cestodes (Moniezia benedeni et Thysaniezia ovilla) et de Cysticercus bovis.

Le Nitroxynil est sans effet sur les Filaires, les Trichures et certains Trichostrongylidés dont Cooperia punctata et Cooperia pectinata.

A la dose d'environ $10 \mathrm{mg} / \mathrm{kg}$ il provoque l'expulsion de Bunostomum phlebotomum et d'Oesophagostomum ( $=$ Bosicola) radiatum, et à celle de $20 \mathrm{mg} / \mathrm{kg}$ d'Haemoncus contortus.

Les doses comprises entre 10 et $20 \mathrm{mg} / \mathrm{kg}$ ont donc des possibilités d'activité qui leur permettent d'intervenir sur Fasciola gigantica et contre quelques Nématodes tels que Bunostomum phlebotomum et Bosicola radiatum, voire Haemoncus contortus.

$\mathrm{Ce}$ registre d'action est cependant moins large que ceux de divers douvicides modernes tels que le dichloro-3, 5-dihydroxy-2-2'-diphényl sulfoxyde (Bitin $S$ ) ou certains dérivés bromés de la salicylanilide (Hilomid).

En milieu tropical où existent trop souvent dans l'organisme des zébus Fasciola gigantica, Paramphistomum, Carmyerius, des Cestodes et des Nématodes, surtout Cooperia et Bosicola, le douvicide à recommander doit être le plus polyvalent possible. L'absence d'action du Nitroxynil sur les Trématodes fréquents dans la panse et sur les grands Cestodes souvent très nombreux dans l'intestin grêle est une lacune qui risque de laisser subsister un parasitisme grave, car il est souvent massif. De ce fait, le Nitroxynil est inférieur à d'autres anthelminthiques et ne peut être, malgré sa facilité d'administration, préconisé en prophylaxie de masse, en Afrique.

Le Nitroxynil administré par voie sous-cutanée agit très rapidement sur les fascioles qui sont tuées assez fréquemment en moins de 72 heures. Certaines d'entre elles demeurent dans la vésicule biliaire où on les recueille diaphanes, transparentes et de couleur vert sale. La plupart du temps elles sont évacuées puis digérées dans l'intestin, sans laisser de traces décelables.

L'existence avant le traitement d'œufs dans les fèces et à l'autopsie la présence de lésions 
plus ou moins importantes des canaux biliaires permettent d'affirmer que l'on est bien en présence d'une fasciolose en cours d'évolution, l'intervention ayant fait disparaître complètement les parasites.

Bosicola radiatum est évacué en 48-72 heures, les Bunostomes et les Haemoncus (intacts dans les fèces, ce qui est rare) en $24-48$ heures.

\section{ESSAIS DE TOXICITE}

Les essais de toxicité ont été effectués à doses progressivement croissantes sur 53 sujets de sexes, d'âges, de poids et d'état d'entretien différents, du mois de janvier 1967 au mois de mai 1969.

Les résultats obtenus sont groupés dans le tableau XXI,

\begin{tabular}{|c|c|c|c|}
\hline $\begin{array}{c}D \underset{(\mathrm{mg} / \mathrm{kg})}{\text { a e }} \mathrm{s} \\
\end{array}$ & $\begin{array}{c}\text { Nombre } \\
\text { d'animaux } \\
\text { uttlisês }\end{array}$ & Mortalitê & Epoque de 1'année \\
\hline $\begin{array}{c}\text { Vole parentérale } \\
10\end{array}$ & 20 & 0 & $\begin{array}{l}\text { Janvier } 1967 \text { - Mai } 1967 \\
\text { Juin } 1969\end{array}$ \\
\hline 15 & 3 & 0 & Juin 1969 \\
\hline 20 & 9 & 0 & Mai 1967 - Mai-Juin 1969 \\
\hline 30 & 3 & 0 & Mai 1967 \\
\hline 40 & 1 & 0 & Avril 1969 \\
\hline 50 & 3 & 2 sur 3 & Mai-Juin 1969 \\
\hline 60 & 2 & 2 sur 2 & Mai 1969 \\
\hline 90 & 1 & 1 & Mai 1969 \\
\hline \multicolumn{4}{|l|}{ Vole orale } \\
\hline 40 & 2 & 0 & Mai 1968 \\
\hline 50 & 1 & 0 & Mai 1969 \\
\hline 60 & 1 & 0 & Juin 1968 \\
\hline 75 & 1 & 0 & Mai 1969 \\
\hline 100 & 4 & 0 & Dëcembre 1968 - Mai 1969 \\
\hline 125 & 2 & 0 & Décembre 1968 - Mai 1969 \\
\hline
\end{tabular}

Il ressort de son examen que l'administration par voie parentérale de doses uniques, faibles, jusqu'à $30 \mathrm{mg} / \mathrm{kg}$ n'ont apparemment aucune conséquence fâcheuse sur la santé de l'animal traité. L'appétit est conservé, mais parfois on constate un ramollissement éphémère des fèces. Une irritation se manifeste autour du point d'inoculation quand le volume du liquide injecté dépasse 10 millilitres, mais elle est le plus souvent fugace. Le médicament paraît s'éliminer assez rapidement et la coloration des tissus est localisée et discrète.

Les premiers signes de l'intoxication, parfois mortelle, sont plus ou moins intenses à partir de $50 \mathrm{mg} / \mathrm{kg}$ sur les animaux de tous âges.
Au-delà de $60 \mathrm{mg} / \mathrm{kg}$, tous les sujets succombent en 2 à 8 heures, selon la dose.

Les zébus présentent des coliques violentes qui durent plusieurs heures; ils se couchent et se relèvent fréquemment. Ils sont couverts de sueur et la respiration est accélérée, bruyante. Les sphincters se relâchent, l'anus est béant et fait entendre un bruit de succion assez caractéristique lorsqu'on entre dans l'étable. Le plus souvent l'appétit est conservé malgré l'intensité des signes cliniques.

A l'autopsie, les organes apparaissent congestionnés et des hémorragies intestinales sont souvent très abondantes. 
Aucune mortalité n'a été observée sur les animaux qui ont absorbé par voie orale une dose unique inférieure à $125 \mathrm{mg} / \mathrm{kg}$.

D'après les faits recueillis, sur 53 zébus traités au Nitroxynil surtout par voie sous-cutanée, il ressort que, pour les doses de $10 \mathrm{mg} / \mathrm{kg}$, l'écart entre la dose thérapeutique et la dose mortelle est de 1 à 5 et à la double dose de $20 \mathrm{mg} / \mathrm{kg}$ il n'est plus que de 1 à 2,5 . Si cette dernière est utilisée, des précautions doivent être prises lorsqu'on intervient sur des animaux bas d'état, en fin de saison sèche (mai - juin juillet).

\section{CONCLUSIONS}

Le Nitroxynil est un anthelminthique dont la polyvalence est limitée à Fasciola gigantica et à quelques "strongles" du tube digestif (Haemoncus, Bunostomum et Bosicola).

Il est sans action sur divers autres Trématodes: Dicrocoelium hospes, les Paramphistomidés et les Gastrothylacidés fréquents dans la panse, Schistosoma bovis, les grands Cestodes parasites de l'intestin et, enfin, sur la plupart des Nématodes, les fillaires des ligaments, des vaisseaux et des séreuses.
Cette polyvalence insuffisante (surtout à l'égard des Paramphistomidés et des diverses espèces de Carmyerius) fait que le médicament ne peut être préconisé dans le traitement de masse des Trématodoses du zébu adulte, en Afrique centrale.

Par contre, il est parfaitement utilisable en Europe où les fascioles sont nombreuses et les Trématodes plus rares dans le rumen.

Le médicament s'injecte sous la peau (solution à $25 \mathrm{p}$. 100) à la dose de $10 \mathrm{mg} / \mathrm{kg}$ pour Fasciola gigantica de plus de 12 semaines et de $20 \mathrm{mg} / \mathrm{kg}$ pour les douves plus jeunes. Dans l'un et l'autre cas, la plupart des " strongles » sensibles à l'anthelminthique sont également chassés.

Le Nitroxynil est généralement bien supporté à la dose de $10 \mathrm{mg} / \mathrm{kg}$. Les premiers accidents mortels apparaissent vers $50 \mathrm{mg} / \mathrm{kg}$ ce qui laisse une marge de sécurité généralement suffisante. A la dose thérapeutique de $20 \mathrm{mg} / \mathrm{kg}$, des précautions sont cependant à prendre.

\footnotetext{
Laboratoire de Parasitologie, Ecole nationale vétérinaire, 94 - Alfort.

Laboratoire de Farcha, Fort-Lamy (République du Tchad).
}

\section{SUMMARY}

Effect of Nitroxynil on different helminths parasites of zebu cattle in Central Africa

In dry tropical Africa, Nitroxynil, in zebu cattle, is an anthelmintic the polyvalence of which is limited to Fasciola gigantica and certain Nematodes of the abomasum and of the intestine (Haemoncus, Bunostomum and Bosicola).

The recommended doses are $10 \mathrm{mg} / \mathrm{kg}$ for Fasciola twelve weeks old and $20 \mathrm{mg} / \mathrm{kg}$ for the youngest ones.

The first fatal accidents arise at $50 \mathrm{mg} / \mathrm{kg}$.

\section{RESUMEN}

\section{Acción del Nitroxynil en varios parásitos del cebú en Africa central}

En las regiones tropicales secas de Africa, el Nitroxynil es. en los cebues, un antihelmíntico cuya acción se limita a Fasciola gigantica y a ciertos nemátodos del abdomen y del intestino (Haemoncus, Bunostomum y Basicola).

Son de $10 \mathrm{mg} / \mathrm{kg}$ las dosis recomendadas contra las Fasciola ya de 12 semanas de edad (de julio a diciembre en Chad) y de $20 \mathrm{mg} / \mathrm{kg}$ contra las formas jovenes.

Los primeros accidentes tóxicos ocurren con $50 \mathrm{mg} / \mathrm{kg}$. 


\section{BIBLIOGRAPHIE}

DAVIS (M.), LUCAS (H.M.S.), ROSENBAUM (J.) GUILHON (J.), "Action du 4-cyano-2-iodo-6-nitroet WRIGHT (D.E.), « 4-cyano-2-iodo-6-nitrophénol: a new fasciolicide», Nature London. 1966, 211, 882 . phénol in vivo sur Fasciola hepatica», C.R. Acad. Sci., Paris, 1966, 263, 1234-1236. 